\title{
Inflammatory bowel disease - is there something new in the immunological background?
}

\author{
Andrzej Marszałek ${ }^{1,2 *}$, Ryszard Marciniak ${ }^{3 *}$, Andrzej Szkaradkiewicz, \\ Agnieszka Wasilewska ${ }^{3}$, Izabela Chudzicka-Strugała ${ }^{4}$, Hanna Ziuziakowska ${ }^{2}$, \\ Małgorzata Żebrowska ${ }^{2}$, Przemysław Majewski ${ }^{1}$, Tomasz Banasiewicz ${ }^{3}$, \\ Michał Drews ${ }^{3}$
}

\author{
${ }^{1}$ Department of Clinical Pathomorphology, Poznan University of Medical Sciences, Poland \\ ${ }^{2}$ Department of Clinical Pathomorphology, Collegium Medicum in Bydgoszcz, \\ Nicolaus Copernicus University in Torun, Poland \\ ${ }^{3}$ Department of General, Gastroenterological and Endocrinological Surgery, \\ Poznan University of Medical Sciences, Poland \\ ${ }^{4}$ Department of Medical Microbiology, Poznan University of Medical Sciences, Poland \\ *Equal contribution of both authors
}

\begin{abstract}
In the present paper we correlate clinical data, as well as histopathological, immunohistochemical and molecular biology methods, with the occurrence of both forms of inflammatory bowel disease (IBD) i.e. ulcerative colitis and Crohn's disease. We found that patients with a history of Epstein-Barr virus (EBV) or cytomegalovirus (CMV) infections, as well as steroid treatment, had increased susceptibility to the development of IBD. The diagnosis of IBD was confirmed by histopathology. Previous infections by EBV and CMV, as well as M. tuberculosis, were proved by PCR-based techniques and in situ hybridization. We found PCR-proved latent viral infections in $30-50 \%$ of the IBD patients we studied. However, we were unable to prove the presence of viral antigens by immunohistochemistry for EBV or CMV. We found positive correlations between the presence of anti-CMV IgG, as well as PCR-positive results for M. tuberculosis with an ulcerative colitis diagnosis. Additionally, up to $80 \%$ of IBD patients used steroids, which was found to be correlated with a diagnosis of Crohn's disease. Our data may support the theory that IBD could be related to previous viral infections and the use of steroids. (Folia Histochemica et Cytobiologica 2011, Vol. 49, No. 2, 357-362)
\end{abstract}

Key words: inflammatory bowel disease, histopathology, EBV, CMV

\section{Introduction}

Idiopathic inflammatory bowel disease (IBD) comprises two major forms: ulcerative colitis (UC) and Crohn's disease (CD). The diagnosis is based on clin-

Correspondence address: A. Marszałek,

Department of Clinical Pathomorphology

Collegium Medicum in Bydgoszcz,

Nicolaus Copernicus University, Torun, Poland,

Sklodowskiej-Curie Str. 9, 85-094 Bydgoszcz, Poland;

tel.: (+ 48 52) 58542 00, fax: (+ 48 52) 58540 49;

e-mail: amars@ump.edu.pl ical, radiological, endoscopic and histopathological studies. However, nowadays about $10-20 \%$ of colectomy specimens remain unclassified and are designated as 'undetermined colitis' [1]. Both diseases are recognized worldwide $[2,3]$. Several attempts have been made to uncover a possible infectious agent as a cause of IBD. But to date, such efforts have failed to provide any suspected pathogen [2-6]. Moreover, several hypotheses, e.g. psychiatric or previous viral infections, on the pathogenesis of IBD have been taken into consideration [3].

The aim of the present study was to estimate if some infections more commonly found in immuno- 
compromised patients, as well as in patients with immune system disregulation, could be correlated with susceptibility to IBD development.

\section{Material and methods}

The study group consisted of 47 patients aged 15-82 years, who underwent bowel surgery in 2005 and 2006 at the Department of General, Gastroenterological and Endocrinological Surgery of the Poznan University of Medical Sciences. There were three study subgroups: 21 patients with UC, 11 patients with $\mathrm{CD}$, and 15 patients with non-IBD partial colectomy or rectum resection, who served as a control group.

All patients were diagnosed using clinical examination and routine histopathological assessment of intestinal specimens. Further histopathological studies as well as immunohistochemical (IHC) studies were performed at the Department of Clinical Pathomorphology in Bydgoszcz.

Microbiological studies were carried out at the Department of Microbiology in Poznan. The study material consisted of swabs and resected intestinal specimens. Additionally, blood serum from IBD patients was obtained. DNA extraction from gut samples was performed by PCR-ELISA technique. Using Mycobacterium paratuberculosis PCR test, Mycobacterium avium subspecies paratuberculosis DNA was evaluated. EBV RNA was assessed using the in situ hybridization technique by evaluation of EBER-1 and EBER-2 molecules. CMV DNA was analyzed by nested-PCR in patients' serum. DNA isolated from a cell culture of a standardized strain was used as a control.

Histopathological assessment. Surgical samples were processed routinely for paraffin blocks and hematoxylin-eosin slides were prepared. Using standard criteria, the diagnosis of CD as well as UC, and other diseases (in controls) was made. Then, using representative (diagnostic) paraffin blocks, we carried out IHC studies for EBV (mouse anti-EBV LMP; clone CS.1-4., DAKO, cat. no. M0897, titer 1:100) and CMV (mouse anti-CMV; clones DDG9 and $\mathrm{CCH} 2$, DAKO, cat. no. N1537, ready-to-use) antigens.

Statistical analysis. Statistical analysis was done using Statistica v. 7.0 software. The normality of distribution of examined parameters was checked by histograms with Gausse curve. None of the examined parameters from studied groups ( $\mathrm{CD}, \mathrm{CU}$, control) had normal distribution. Correlation matrices analysis were employed to assess the correlation between examined parameters. For data analysis, we used correlation matrices. For statistical significance, $\mathrm{p}<$ $<0.05$ was assumed.

\section{Results and discussion}

Analysis of duration of the disease showed average time up to 12 years of clinical history, before intestinal resection in the $\mathrm{CD}$ group. In other groups, aver- age length of clinical history was: UC 5.8 years, control group 0.7 years (Table 1 ). We found a history of tuberculosis in two UC patients (9.5\%), three patients $(27.3 \%)$ with $\mathrm{CD}$ and one $(6.7 \%)$ in the control group. A history of mumps was recorded in four patients $(19 \%)$ in the UC group, three $(27.3 \%)$ in the CD group and five patients $(33.3 \%)$ from the control group. The frequency of measles in the studied groups was $38.1 \%, 54.5 \%$ and $40 \%$, in UC, and CD and controls, respectively (Table 1 ).

A history of the use of steroids or other immunosuppressive drugs was recorded only in UC and CD patients. A history of previous acute streptococcal pharyngitis was found in ten UC patients (47.6\%), in six CD patients $(54.5 \%)$ but only in three $(20 \%)$ cases from the control group.

Diarrhea was present in all patients with UC, while blood in stool was found in $81 \%$. The highest prevalence of ileus $(63.6 \%)$ was in CD patients. In $66.7 \%$ of the control group we found blood in patients' stools. There were 11 emergency operations performed: six in patients with UC $(28.6 \%)$ and five in patients with CD (45.5\%); 36 operations were planned ahead of time, including 15 in patients with UC $(71.4 \%)$, six with CD (54.5\%), and all $15(100 \%)$ cases in the control group. The localization of changes in the bowel was: colon in $85.7 \%$ of the UC group, small bowel in $45.5 \%$ of CD patients, and rectum in $66.7 \%$ of the control group. Details of patients' characteristics are presented in Table 1.

Some recent reports have suggested that initiation of IBD is somehow obscure. However, cells of innate immunity produce cytokines essential for inflammatory reactions, as well as factors critical for the subsequent initiation of specific immunity. However, such data has been not consistently reproduced. In the present study, a clinical diagnosis of IBD, and a diagnosis of other bowel diseases, was confirmed by double independent pathological opinion in all studied patients. All cases in the CD and UC groups fulfilled the morphological criteria of IBD (Figure 1). Additional studies revealed Mycobacterium avium subspecies paratuberculosis (MASP), with the highest percentage of positive results in the CD group - seven $(63.6 \%)$ (Table 2). There were no laboratory or clinical features of recent CMV infection (anti-CMV IgM was negative in all groups). But previous exposure to viral antigens, expressed by presence of anti-CMV $\mathrm{IgG}$, was confirmed in $25 \%$ of patients with $\mathrm{CD}$, and in more than $40 \%$ of both UC and controls. Using molecular biology tests, viral nucleic acids were found in some patients.

For CMV, the percentage of positive results was lower in $\mathrm{CD}$ and higher in both $\mathrm{UC}$ and control groups. For EBV, the lowest number of positive pa- 
Table 1. Patients' general clinical characteristics

\begin{tabular}{|c|c|c|c|}
\hline \multirow[b]{2}{*}{ Data } & \multicolumn{3}{|c|}{ Group } \\
\hline & UC & CD & Control \\
\hline Gender & $11 \mathrm{~F} / 10 \mathrm{M}$ & $7 \mathrm{~F} / 4 \mathrm{M}$ & $10 \mathrm{~F} / 5 \mathrm{M}$ \\
\hline Age in years (range) & $39.7(15-60)$ & $41.4(20-82)$ & $60.7(40-79)$ \\
\hline Disease duration (years) & $5.8(0.5-25)$ & $12.0(0.5-34)$ & $0.7(0.5-1.0)$ \\
\hline $\begin{array}{l}\text { Clinical history } \\
\text { Tuberculosis } \\
\text { Mumps } \\
\text { Measles } \\
\text { Acute streptococcal pharyngitis } \\
\text { Use of steroids } \\
\text { Use of other immunosuppressive drugs }\end{array}$ & $\begin{array}{c}2(9.5 \%) \\
4(19 \%) \\
8(38.1 \%) \\
10(47.6 \%) \\
15(71.4 \%) \\
4(19 \%)\end{array}$ & $\begin{array}{l}3(27.3 \%) \\
3(27.3 \%) \\
6(54.5 \%) \\
6(54.5 \%) \\
9(81.8 \%) \\
1(9.1 \%)\end{array}$ & $\begin{array}{c}1(6.7 \%) \\
5(33.3 \%) \\
6(40 \%) \\
3(20 \%) \\
0 \\
0\end{array}$ \\
\hline $\begin{array}{l}\text { Disease localization } \\
\text { Colon } \\
\text { Rectum } \\
\text { Jejunum/cecum } \\
\text { Small bowel }\end{array}$ & $\begin{array}{c}18(85.7 \%) \\
2(9.5 \%) \\
0 \\
1(4.8 \%)\end{array}$ & $\begin{array}{c}1(9.1 \%) \\
1(9.1 \%) \\
4(36.4 \%) \\
5(45.5 \%)\end{array}$ & $\begin{array}{c}5(33.3 \%) \\
10(66.7 \%) \\
0 \\
0\end{array}$ \\
\hline $\begin{array}{l}\text { Symptoms } \\
\text { Diarrhea } \\
\text { Blood in stool } \\
\text { Toxic megacolon } \\
\text { Obstruction } \\
\text { Inflammatory tumor } \\
\text { Intestinal fistula }\end{array}$ & $\begin{array}{c}21(100 \%) \\
17(81 \%) \\
1(4.8 \%) \\
2(9.5 \%) \\
0 \\
0\end{array}$ & $\begin{array}{c}1(9.1 \%) \\
0 \\
0 \\
7(63.6 \%) \\
5(45.5 \%) \\
2(18.2 \%)\end{array}$ & $\begin{array}{c}0 \\
10(66.7 \%) \\
0 \\
0 \\
0 \\
0\end{array}$ \\
\hline $\begin{array}{l}\text { Surgery } \\
\text { Emergency } \\
\text { Planned }\end{array}$ & $\begin{array}{c}6(28.6 \%) \\
15(71.4 \%)\end{array}$ & $\begin{array}{l}5(45.5 \%) \\
6(54.5 \%)\end{array}$ & $\begin{array}{c}0 \\
15(100 \%)\end{array}$ \\
\hline
\end{tabular}

UC - ulcerative colitis; CD — Crohn's disease; F — female; M - male

tients was found in the UC group and higher values were found in $\mathrm{CD}$ and controls (Table 2). However, IHC for viral antigens for EBV or CMV were negative (Figure 2).

Nowadays, one theory of IBD development is based on a 'dysbiosis' or 'disregulated' immune response leading to intestinal disease by altered innate and adaptive immunity with unlimited activation of immunological mechanisms of inflammation [7].

It has been studied in animal and clinical models [7]. Such models have revealed abnormal expression of heat shock proteins (Hsp), cytokines, chemokines, growth factors as well as TNF and eicosanoids and other bioactive proteins in IBD cases [8]. But it remains an open question as to what else could be a potent predisposing factor to IBD development.

Powell et al. first reported a patient with UC and cytomegalic inclusion disease in 1961 [for review see 9]. According to recent epidemiological data, patients with severe steroid-resistant UC might be at particular risk for CMV infection [10-13]. In our studies, anti-CMV IgG was confirmed in $41.7 \%$ and CMV DNA was positive in the serum of $53.3 \%$ of control patients aged $40-79$. The reactivation of CMV is a consequence of cell-mediated impaired immunity
[14]. In some patients, CMV may coexist with other infectious agents (e.g. Cryptosporidium spp., Isospora belli, Herpes simplex types 1 and 2, Mycobacterium avium-intracellular) found within the bowel $[9,10,12,15]$. According to previously published data, CMV infection was detected fairly frequently $(21 \%)$ in the surgical specimens of UC, and such cases were steroid resistant $[9,15]$. On the other hand, patients with severe, steroid-refractory and steroid-dependent IBD are usually treated with immunosuppressive agents, and such therapy might increase the risk of CMV infection [10].

In a retrospective, case-controlled study, $\mathrm{CMV}$ was detected by immunohistochemistry only in ten of 40 $(25 \%)$ patients with refractory UC and in $2.5 \%$ of patients with non-refractory UC [16]. Other authors have suggested that the prevalence of CMV complicated UC is less common then severe steroid-refractive $\mathrm{CD}$ with $\mathrm{CMV}$ positive patients estimated between $11 \%$ and $36 \%$ of cases [17]. In our study, serum CMV DNA was found in nine $(42.9 \%)$ UC patients and four $(36.4 \%) \mathrm{CD}$ patients. There were no laboratory or clinical features of recent CMV infection in our IBD cases. Anti-CMV IgM was negative in all groups. Anti-CMV IgG was confirmed in sev- 
en $(41.2 \%)$ UC patients and two (25\%) CD patients. Although in some cases we were able to prove a previous CMV infection by laboratory test or molecular biology techniques, we were unable to prove the presence of CMV antigens in the removed intestine nor in the local lymph nodes. Such findings support aforementioned suggestions [18]. However, it re-

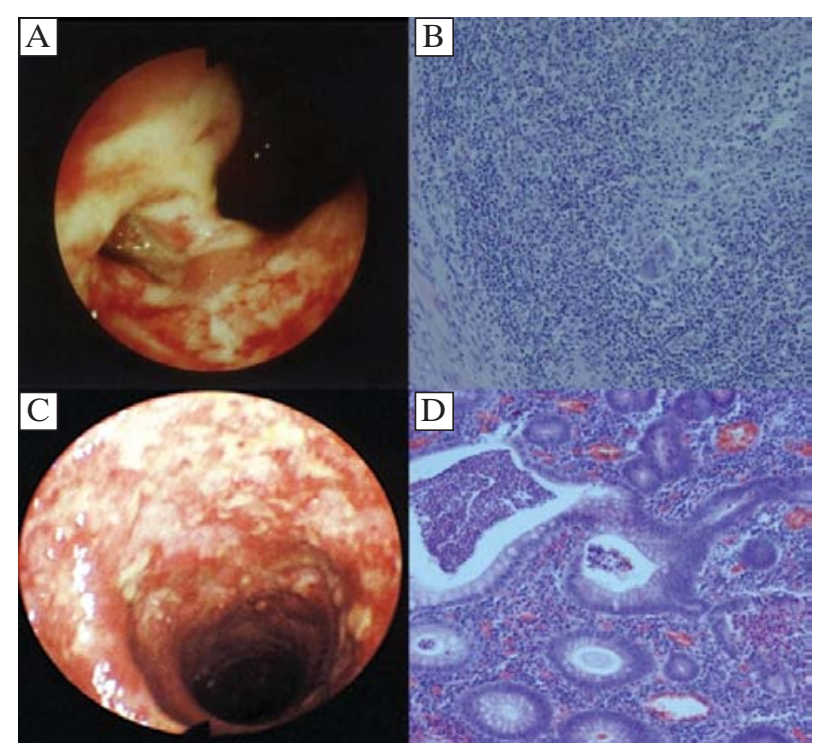

Figure 1. Inflammatory bowel disease morphological presentation. A. Endoscopic picture of changed mucosa in Crohn's disease; B. Sarcoid-like granuloma within the wall of large bowel; C. Macroscopic view of colon ulcerative colitis; D. Destruction of colonic crypts as well as cryptal abscesses easily visible in tissue sample from patient with ulcerative colitis. Photographs B and D, hematoxylin-eosin slides, primary objective magnification $\times 10$ mains unclear whether CMV infection has an active role in IBD development, or whether it exists coincidentally $[11,15]$.

Spieker et al. suggested that productively and latently EBV-infected cells are detectable in many, but not all, cases of IBD, and are more frequently detectable in active UC than in CD or controls [19]. In our study, there were no clinical or histological features of EBV infection in studied cases. Previously published data on IBD patients has shown EBV latent or productive infection in tonsils but not in any intestinal epithelial cells [20]. The results of our study confirm that observation. We found that in the UC group patients were EBV positive in $35.8 \%$, with $54.5 \%$ in $\mathrm{CD}$ cases. However, in IHC studies, the presence of viral antigens in intestine samples for EBV was negative. Overall, EBV is unlikely to play a role as an etiological agent in IBD.

Using clinical data and the results of laboratory tests, the following correlations were found. In the CD group, the history of previous acute Streptococcal pharyngitis correlated with a history of measles. Patients with a clinical presentation of disease as obstruction had no previous history of mumps, while those who suffered from vesico-urinary fistula tended to have mumps in their clinical record. Patients with UC tended to have a history of measles and mumps as well as acute Streptococcal pharyngitis. Such a tendency was also observed in controls, but not in CD patients. Moreover, we found that in UC and in controls, but not in the CD group, there was a correlation between features of CMV infection, MASP and measles infection. CD patients, distinctively, were found to be those with important steroid

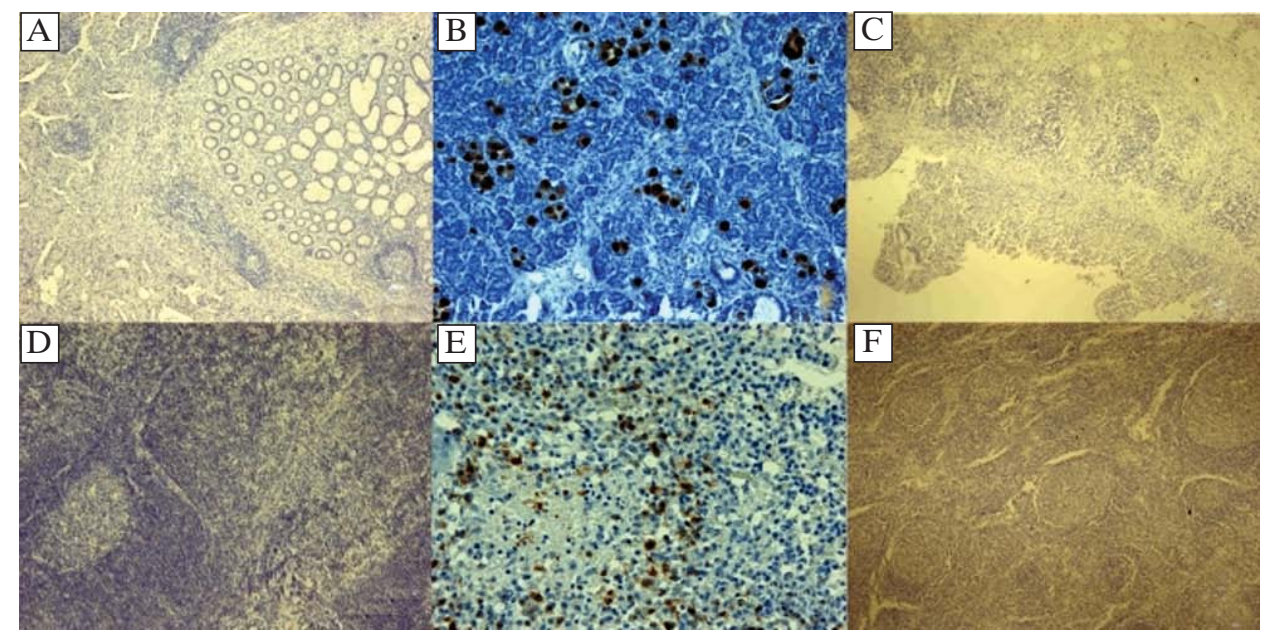

Figure 2. Immunohistochemical studies on presence of CMV (A, D) and EBV $(\mathbf{C}, \mathbf{F})$ antigens in tissue samples from patients with inflammatory bowel disease in whom molecular studies revealed viral nucleic acids. No positive reaction visible. Positive controls for CMV (B) and for EBV (E) done on tissue samples from fatal cases of either EBV or CMV congenital infection. In all photographs, primary objective magnification $\times 10$ 
Table 2. Results of molecular tests for presence of viral infections in studied patients

\begin{tabular}{|l|c|c|c|}
\hline & UC & CD & Control \\
\hline MASP & $6(28.6 \%)$ & $7(63.6 \%)$ & $6(40 \%)$ \\
\hline CMV & $9(42.9 \%)$ & $4(36.4 \%)$ & $8(53.3 \%)$ \\
\hline anti-CMV IgM & 0 & 0 & 0 \\
\hline anti-CMV IgG & $7(41.2 \%)$ & $2(25 \%)$ & $5(41.7 \%)$ \\
\hline EBV & $5(35.8 \%)$ & $6(54.5 \%)$ & $7(58.3 \%)$ \\
\hline
\end{tabular}

CMV — cytomegalovirus; EBV — Epstein-Barr virus; MASP — Mycobacterium avium subspecies paratuberculosis; UC — ulcerative colitis; $\mathrm{CD}$ - Crohn's disease
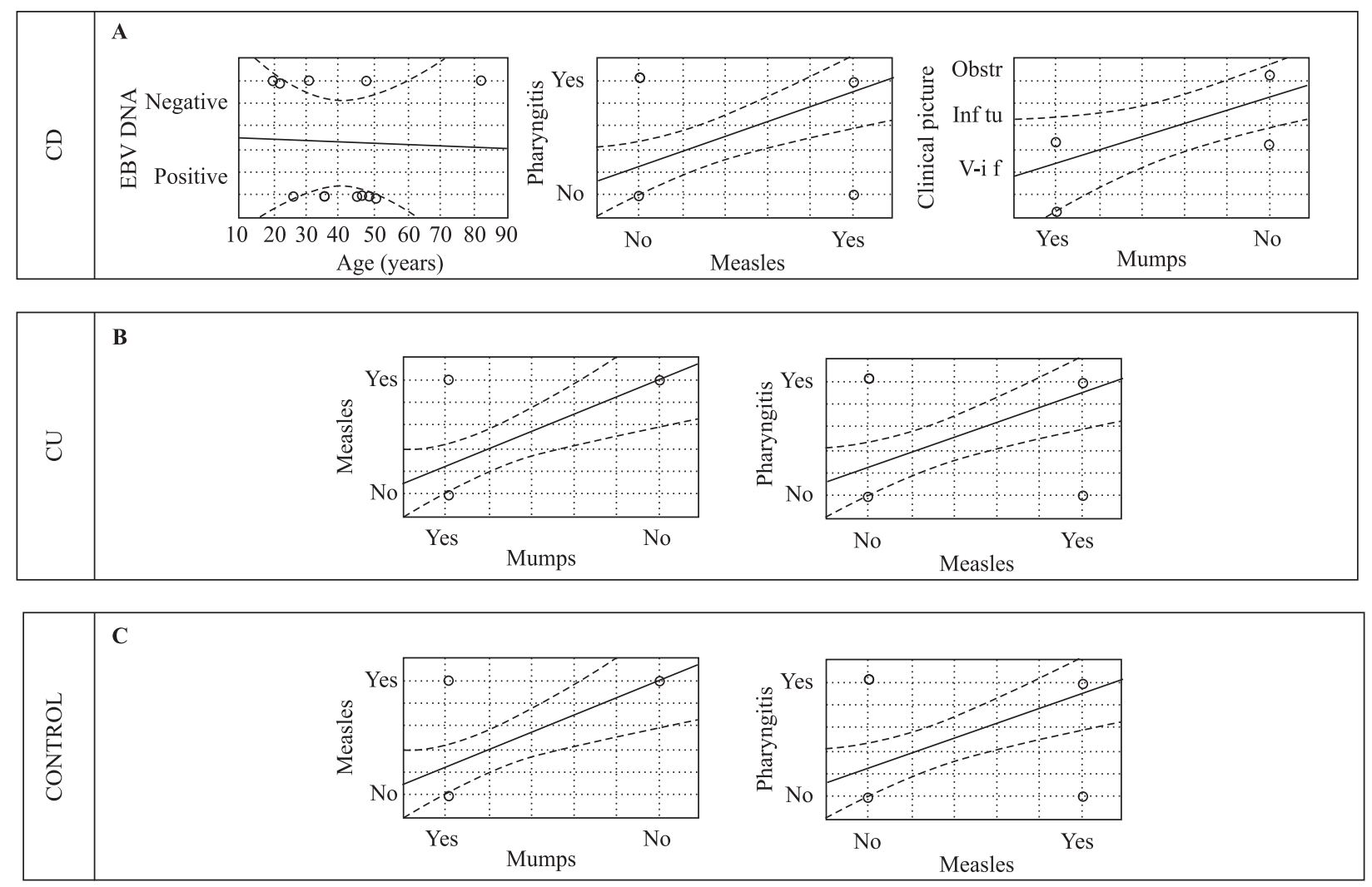

\begin{tabular}{|l|c|c|c|c|c|c|c|c|}
\hline \multirow{2}{*}{$\mathbf{d}$} & \multicolumn{2}{|c|}{ CD } & \multicolumn{3}{c|}{ UC } & \multicolumn{3}{c|}{ CONTROL } \\
\cline { 2 - 9 } & Mumps & Steroids & Measles & MASP & CMV DNA & Measles & MASP & CMV DNA \\
\hline Location & NS & $\mathrm{p}<0.01$ & $\mathrm{p}=0.04$ & NS & NS & $\mathrm{p}<0.04$ & NS & NS \\
\hline CMV DNA & NS & NS & NS & $\mathrm{p}<0.01$ & NS & NS & $\mathrm{p}<0.01$ & NS \\
\hline IgG-anty-CMV & NS & NS & NS & $\mathrm{p}<0.01$ & $\mathrm{p}=0.0001$ & NS & $\mathrm{p}<0.01$ & $\mathrm{p}=0.001$ \\
\hline Type of surgery & $\mathrm{p}<0.03$ & NS & NS & NS & NS & NS & NS & NS \\
\hline
\end{tabular}

Figure 3. Correlations between selected clinical data and laboratory studies results

CMV — cytomegalovirus; EBV — Epstein-Barr virus; MASP — Mycobacterium avium subspecies paratuberculosis; obstr — obstruction; inf tu — inflammatory tumor; v-i f — vesico-intestinal fistula; NS — not significant; UC — ulcerative colitis; CD — Crohn's disease

use in their clinical history, as well as a history of mumps. These patients had also, according to their disease, different types of surgery. Details are presented in Figure 3.
Cellular infiltrate in IBD reflects incorrect orchestration of plethora of different stimulators of inflammatory response. It is reasonable to suspect that those patients might also present other abnormalities in 
inflammatory reaction. Among such, there are more common viral infections in past history, as was proved in the present study. Specific immunologic alterations in IBD were attributed to NOD2/CARD15 mutations $[3,21]$. Abnormal function of NOD2 might lead to altered bacterial killing and be eventually directly related to mucosal damage [22]. Moreover, recently additional indirect influence of mutation in the NOD2 gene through cytokine production has been postulated [22]. There were also described roles of TLR4 genes polymorphism and additionally such factors as IL-23R, cytokines (INF- $\gamma$, TNF- $\alpha$, TGF- $\beta$, IL-4, IL-5, IL-6, IL-12, IL-13, IL-17, IL-21, IL-22, IL-26, IL-27, IL-31, IL-32), local microflora, bacterial infection, tobacco smoking, oral contraceptives, and even mucosal integrity [21, 22]. All these factors play important roles in driving an inappropriate response of T cells (especially Th1, 2, and 17) to possible enterocolic infectious agent. An inadequately inhibited reaction leads to full-blown IBD. Recently, the number of molecules believed to be potentially important in IBD development has increased dramatically. It now includes IL-1, IL-2R, CAMS, addressins, defensins, flagellins, granulysins, selectins, claudins, annexins, guanilyns, laminins, intimins, aquaporins, microsins, adaptins, fibrillarins, syndecam-1, stromelysin, integrins, galanin-1, tropomyosin, FGF, EGF, permeability-enchancing factor, neurotrophins, survivins, ubiquitins and zonulins [3, 21].

To sum up, we should look for a better understanding of polygenic and multifactor etiology of IBD, for instance at impaired patients' immunity, e.g. expressed by higher susceptibility to some fairly common infections or need for steroid therapy, as reflected in the history of our patients.

\section{References}

1. Geboes K. Indeterminate colitis: a review of concept - what's in a name? Inflamm Bowel Dis. 2008;14:850-857.

2. Dimitroulia E. Frequent detection of cytomegalovirus in the intestine of patients with inflammatory bowel disease. In flamm Bowel Dis. 2006;12:879-884.

3. Kirsner JB. Inflammatory bowel disease: from the mystical to the cellular and now the molecular. World $J$ Gastroenterol. 2005;26:4127-4128.

4. Bernstein CN. Testing the interaction between NOD-2 status and serological response to Mycobacterium paratubercu- losis in cases of inflammatory bowel disease. J Clin Microbiol. 2007;45:968-971.

5. Gophna U. Differences between tissue-associated intestinal microfloras of patients with Crohn's disease and ulcerative colitis. J Clin Microbiol. 2006;44:4136-4141.

6. Swidsinski A. Spatial organization and composition of the mucosal flora in patients with inflammatory bowel disease. J Clin Microbiol. 2005;43:3380-3389.

7. Tlaskalova-Hogenova H. Commensal bacteria (normal microflora), mucosal intensity and chronic inflammatory and autoimmune disease. Immunol Lett. 2004;93:97-108.

8. Sepulveda S. Inflammatory bowel diseases: an immunological approach. Rev Med Chile. 2008;136:367-375.

9. Galiatsatos P. Meta-analysis of outcome of cytomegalovirus colitis in immunocompetent hosts. Dig Dis Sci. 2005; 50:609-616.

10. Minami M. Cytomegalovirus infection in severe ulcerative colitis patients undergoing continuous intravenous cyclosporine treatment in Japan. World J Gastroenterol. 2007; 13:754-760.

11. Kuwabara A. Clinicopathologic characteristics of clinically relevant cytomegalovirus infection in inflammatory bowel disease. J Gastroenterol. 2007;42:823-829.

12. Roskell DE. HIV associated cytomegalovirus colitis as a mimic of inflammatory bowel disease. Gut. 1995;37:148-150.

13. Takahashi Y. Prevalence of cytomegalovirus infection in inflammatory bowel disease patients. Dis Colon Rectum. 2004:47:722-726.

14. Jaber S. Cytomegalovirus infection in critically ill patients: associated factors and consequences. Chest. 2005;127:233-241.

15. Kishore J. Infection with cytomegalovirus in patients with inflammatory bowel disease: prevalence, clinical significance and outcome. J Med Microbiol. 2004;53:1155-1160.

16. Inoue $\mathrm{T}$. Refractory ulcerative colitis accompanied with cytomegalovirus colitis and multiple liver abscesses: a case report. World J Gastroenterol. 2005;33:5241-5244.

17. Olsen S. Cytomegalovirus infection in Crohn's colitis. $J R$ Soc Med. 2004;97:335-336.

18. Criscuoli V. Cytomegalovirus and inflammatory bowel disease: is there a link? World J Gastroenterol. 2006; 30:4813-4818.

19. Spieker T. Distribution and phenotype of Epstein-Barr virusinfected cells in inflammatory bowel disease. Am J Pathol. 2000;157:51-57.

20. Anagnostopoulos I. Morphology, immunophenotype and distribution of latently and/or productively Epstein-Barr virus-infected cells in acute infectious mononucleosis: implications for the interindividual infection route of Epstein-Barr virus. Blood. 1995;85:744-750.

21. Jaber S. Cytomegalovirus infection in critically ill patients: associated factors and consequences. Chest. 2005;127:233-241.

22. Andoh A. Mucosal cytokine network in inflammatory bowel disease. World J Gastroenterol. 2008;33:5154-5161.

Submitted: 12 July, 2010

Accepted after reviews: 4 February, 2011 